\title{
A formação de egressos de Enfermagem e seus estranhamentos no mundo do
}

\section{trabalho em saúde}

\author{
The training of Nursing graduates and their strangeness in the world of health work \\ La formación de los egresados de Enfermería y sus extrañamientos en el mundo del trabajo en \\ salud
}

\author{
Shino Shoji \\ ORCID: https://orcid.org/0000-0001-8987-2291 \\ Universidade Federal do Rio de Janeiro, Brasil \\ E-mail: shinoshoji@gmail.com \\ Djynnana de Azevedo Avena \\ ORCID: https://orcid.org/0000-0001-6130-5551 \\ Petrobras Petróleo Brasileiro, Brasil \\ E-mail: djynnana@yahoo.com.br \\ Eloá Carneiro Carvalho \\ ORCID: https://orcid.org/0000-0002-1099-370X \\ Universidade do Estado do Rio de Janeiro, Brasil \\ E-mail: eloagrossi@uol.com.br \\ Samira Silva Santos Soares \\ ORCID: https://orcid.org/0000-0001-9133-7044 \\ Universidade Federal do Rio de Janeiro, Brasil \\ E-mail: samira_opg@hotmail.com \\ Thereza Christina Mó y Mó Loureiro Varella \\ ORCID: https://orcid.org/0000-0001-9389-1161 \\ Universidade do Estado do Rio de Janeiro, Brasil \\ E-mail: thereza1208@gmail.com \\ Sheila Nascimento Pereira de Farias \\ ORCID: https://orcid.org/0000-0001-5752-265X \\ Universidade Federal do Rio de Janeiro, Brasil \\ E-mail: sheilaguadagnini@gmail.com \\ Karla Biancha Silva de Andrade \\ ORCID: https://orcid.org/0000-0002-6216-484X \\ Universidade do Estado do Rio de Janeiro, Brasil \\ E-mail: karla.biancha@gmail.com \\ Sandra Regina Maciqueira Pereira \\ ORCID: https://orcid.org/0000-0002-0550-2494 \\ Universidade do Estado do Rio de Janeiro, Brasil \\ E-mail: sandregina@gmail.com \\ Norma Valéria Dantas de Oliveira Souza \\ ORCID: https://orcid.org/0000-0002-2936-3468 \\ Universidade do Estado do Rio de Janeiro, Brasil \\ E-mail:norval_souza@yahoo.com.br
}

\begin{abstract}
Resumo
Objetivo: Analisar o impacto das ações acadêmico-pedagógicas na formação dos estudantes de enfermagem para atuação no mundo do trabalho. Método: Tratou-se de estudo qualitativo, desenvolvido em duas universidades federais no Rio de Janeiro. Participaram 43 egressos, aos quais se aplicou um roteiro de entrevista semiestruturada, entre junho e setembro de 2016. Para o tratamento dos dados, optou-se pelo emprego da análise lexical/textual, por meio da utilização do software IRAMUTEQ (Interface de $R$ pour les Analyses Multidimensionnelles de Textes et de Questionnaires), permitindo dois tipos de interpretação de resultados: frequência e rede de palavras. Resultados: Captaram-se duas temáticas principais: Vivências práticas e o protagonismo do curso de residência; e Fragilidades da formação. Conclusão: Verificou-se que a relação estabelecida entre a teoria e a prática precisa ser fortalecida, devendo acontecer de maneira articulada, através de sucessivas aproximações com a realidade laboral, indo num crescente de vivências que permitam aprofundar as experiências do que é ser enfermeiro.
\end{abstract}

Palavras-chave: Educação em enfermagem; Estudantes; Currículo; Trabalho.

\section{Abstract}

Objective: To analyze the impact of academic-pedagogical actions on the formation of Nursing students to act in the world of work. Method: This was a qualitative study, conducted at two federal universities in Rio de Janeiro. Forty- 
three graduates participated, and a semistructured interview script was applied to them from June to September 2016. For data processing, we opted for lexical/textual analysis, using the software IRAMUTEQ (Interface de $R$ pour les Analyses Multidimensionnelles de Textes et de Questionnaires), allowing two types of interpretation of results: frequency and word network. Results: Two themes were captured: practical experiences and the protagonism of the residency course; and weaknesses of the formation. Conclusion: It was found that the theory-practice relationship needs to be strengthened, and must happen in an articulated manner, through successive approximations with the working reality, going through a growing experience that allows deepening the experiences of being a nurse.

Keywords: Nursing education; Students; Curriculum; Work.

\section{Resumen}

Objetivo: Analizar el impacto de las acciones académico-pedagógicas en la formación de los estudiantes de enfermería para actuar en el mundo laboral. Método: Estudio cualitativo, desarrollado en dos universidades federales en Río de Janeiro. El estudio contó con la participación 43 egresados, a los que se aplicó un guion de entrevista semiestructurada, entre junio y septiembre de 2016. Se decidió emplear el análisis léxico/textual para tratar los datos, utilizando el software IRAMUTEQ (Interface de R pour les Analyses Multidimensionnelles de Textes et de Questionnaires), permitiendo dos tipos de interpretación de resultados: la frecuencia y la red de palabras. Resultados: Se capturaron dos temas: Experiencias prácticas y el protagonismo del curso de residencia; y Debilidades de la formación. Conclusión: Se encontró que la relación teoría-práctica necesita ser fortalecida, y debe ocurrir de manera articulada, a través de sucesivos abordajes con la realidad laboral, entrando en un número creciente de vivencias que permitan profundizar las experiencias de lo que es ser enfermero.

Palabras clave: Educación en enfermería; Estudiantes; Currículo; Trabajo.

\section{Introdução}

Atualmente, o desafio da enfermagem reside na qualidade da formação profissional oferecida e na forma como esta impacta na atuação dos egressos no complexo mundo do trabalho em saúde. Compreender essa problemática é desvendar os inúmeros fatores que perpassam o tema, trazendo-os para um espaço de discussão aprofundado e crítico. Neste sentido, as instituições educacionais têm sido pressionadas em algumas direções, seja no caminho de uma produção contínua e maciça de conhecimento e de tecnologia, ou ainda, na formação de profissionais cada vez mais capacitados para exercerem a polivalência e multifuncionalidade que permeia o campo da saúde. Assim, preparar o futuro enfermeiro para esse mundo intricado do trabalho não é tarefa simples e trivial (Silva et al., 2019; Püschel et al, 2017).

As transformações mundiais decorrentes do desenvolvimento científico, econômico e tecnológico têm provocado mudanças importantes em todos os contextos sociais, e consequentemente, nos hábitos, valores, prioridades e na própria visão do eu, do outro e do mundo, impactando sobremaneira no contexto do trabalho em saúde. Isso tem exigido constante revisão das regras de convivência social, das práticas profissionais e da educação, em um ambiente permeado pela atualização acelerada e contínua, além da recusa à simplificação e à fragmentação do saber.

A formação e atuação de enfermeiros têm sido objeto frequente de estudos e práticas diferenciais de ensino. De um modo geral, aponta-se para a relação existente entre ensino, sociedade e mercado de trabalho, e como as interfaces estabelecidas entre estes três elementos têm contribuído para o planejamento e a gestão dos processos de formação. Nessa perspectiva, buscase identificar formas, métodos e conteúdo que devem ser assumidos pelo ensino superior, melhorando o desenvolvimento social, econômico e cultural, segundo as necessidades dos usuários, de um ponto de vista cada vez mais amplo (Silva, Peres, Pio, Marin, \& Otani, 2019; Fettermann et al., 2018).

As discussões sobre a profissionalização dos enfermeiros incidem sobre a identidade profissional, a função social desta categoria profissional e a articulação com as demais áreas do conhecimento, além da necessidade de diversificação teórica e de flexibilização do currículo. Essas perspectivas são desafios para o desenvolvimento das competências exigidas na formação profissional. Assim, os cursos de graduação em enfermagem têm buscado sua inserção nesse cenário complexo e em constante transformação, instrumentalizando os futuros enfermeiros com um perfil generalista, técnico, científico e humanista, com capacidade crítica e reflexiva, preparados para atuar em diferentes níveis de atenção do processo saúde-doença, pautando-se em princípios ético (Silva et al., 2019; Alves \& Silva, 2019). 
Para as escolas de enfermagem, o grande desafio é aproximar o processo de formação das inovações decorrentes das diretrizes curriculares e profissionais, de modo a qualificar o enfermeiro para uma atuação de competência no atual contexto político-econômico, cultural e de saúde do país (Costa, Francisco, \& Hamamoto, 2019). Isto precisa funcionar como ponto de partida de um projeto maior, que traz à tona este debate, pois se compreende que a educação é uma área dinâmica do conhecimento, que dá margem a múltiplos olhares, e distintas compreensões e interpretações. Conscientes das responsabilidades sociais como enfermeiros e cidadãos, faz-se necessário o comprometimento em permanecer vigilantes e atuantes na luta por uma educação construtiva, libertadora, emancipadora, inclusiva e solidária, que tenha como objetivo maior a construção de um alicerce de formação de profissionais de enfermagem capazes de atender à demanda social e de interferir no processo saúdedoença de forma competente, ética e sensível. Nesse sentido, vale-se do ordenamento jurídico brasileiro vigente, dos princípios do Sistema Único de Saúde (SUS) e das Diretrizes Curriculares Nacionais (DCN), além dos aspectos éticos e legais do exercício da profissão.

Dessa forma, buscando contribuir com a melhoria do cenário de formação profissional do enfermeiro e as repercussões de sua atuação no mundo do trabalho, objetivou-se descrever e analisar os impactos do distanciamento entre as ações acadêmicopedagógicas vivenciadas na formação dos estudantes de enfermagem e a realidade encontrada nos serviços de saúde.

\section{Metodologia}

Trata-se de um estudo de natureza qualitativa, com abordagem exploratória e descritiva, realizada em duas faculdades de enfermagem de caráter federal, ambas localizadas no Rio de Janeiro, classificadas como Universidade A e Universidade B. Utilizou-se tal codificação com o intuito de respeitar a confidencialidade das mesmas.

Os participantes foram egressos dessas universidades, elencando-se como critérios de inclusão no estudo: a) enfermeiros que trabalhassem na profissão há pelo menos um ano e b) graduados em enfermagem durante o recorte temporal de 2000 a 2013. Considerou-se relevante a atuação na enfermagem por pelo menos um ano, pois este é um tempo suficiente para se apreender os meandros do mundo do trabalho e do processo laboral. Também se determinou o recorte temporal de 2000-2013 por entender que foi uma década de profundas transformações no contexto do trabalho, com emprego maciço de tecnologias diferenciadas e aplicabilidade de princípios neoliberais para aumento da produtividade e dos ganhos econômicos e financeiros.

Como critério de exclusão dos participantes, adotou-se: enfermeiros que não estivessem atuando no Brasil, pois o foco do estudo centrou-se no mundo do trabalho brasileiro.

Sobre o quantitativo de participantes, planejou-se coletar dados com um graduado de cada turma do referido recorte temporal, perfazendo um total de 56 enfermeiros, ou seja, quatro egressos por ano. A escolha dos participantes foi aleatória, feita por meio de sorteio. Ao final do processo de coleta, obteve-se um total de 43 participantes, sendo 21 egressos da Universidade A e 22 egressos da Universidade B. Essa quantidade reduzida, em relação ao planejado inicialmente, justificou-se pela falta de dados cadastrais dos egressos, recusas de participação no estudo e falta de retorno aos convites de participação. Desse modo, no ano de 2001 não houve representatividade de egressos de ambas as universidades. Os demais anos foram representados por pelo menos um egresso de cada instituição.

O instrumento de coleta utilizado foi uma entrevista semiestruturada, aplicada no período de junho a setembro de 2016. Por tratar-se de um recorte de uma tese de doutorado, esse estudo abordou pontualmente o questionamento feito aos egressos quanto à formação na graduação ter sido suficiente para o preparo do contexto do mundo do trabalho em saúde. Ressalta-se que se recorreu às redes sociais virtuais como fonte alternativa de busca para possíveis participantes da pesquisa, elegendo-se o Facebook, o LinkedIn e o WhatsApp para captar os participantes, bem como a Plataforma Lattes.

Para o tratamento dos dados coletados nas entrevistas, optou-se pela análise lexical ou textual. Foram aplicados métodos estatísticos aos materiais verbais transcritos, permitindo explorações minuciosas e viabilizando a construção de categorias 
naturais a partir de algumas técnicas estatísticas no campo de dados qualitativos (Justo \& Camargo, 2014). Esse tipo de análise tem como unidade o vocabulário: as palavras são identificadas e quantificadas em termos de frequência, priorizando, assim, a sistematização dos dados textuais, para somente depois interpretá-los (Kami et al., 2016).

Fundamentando-se nessa perspectiva, após a transcrição individual do conteúdo de cada entrevista, realizou-se a análise textual com o auxílio do software denominado IRAMUTEQ (Interface de R pour les Analyses Multidimensionnelles de Textes et de Questionnaires), que é um programa livre ancorado no software $R$ (pacote gratuito para realizar análises estatísticas), que possibilita diferentes processamentos e análises estatísticas dos textos inseridos (Zhou et al., 2015). Esse software permitiu dois tipos de interpretação de resultados para esta pesquisa: frequência e rede de palavras.

A frequência de palavras, ou lexicografia básica, é um indicador que apresenta a contagem das palavras presentes no texto analisado. Nesta etapa, são observadas as palavras mais frequentes no texto, deduzindo que determinado vocábulo frequentemente repetido seja pertinente no contexto investigado (Souza, Wall, Thuler, Lowen, \& Peres, 2018) e que sejam encontrados itens passíveis de categorização. Os dados foram então submetidos ao software IRAMUTEQ e em seguida tratados à luz da análise temática de conteúdo. Emergiu daí a categoria "Contexto do trabalho em saúde: aspectos facilitadores e limitadores da atuação dos egressos".

Atendendo aos preceitos éticos, esta pesquisa foi cadastrada na Plataforma Brasil do Ministério da Saúde, conforme preconizado pela Resolução n. ${ }^{\circ}$ 466/2012, do Conselho Nacional de Saúde (CNS/MS), obtendo aprovação do Comitê de Ética sob protocolo $\mathrm{n}^{\mathrm{o}}$ 1.293.828, e Certificado de Apresentação para Apreciação Ética em Pesquisa (CAAE) sob o $\mathrm{n}^{\circ}$ 49971815.5.0000.5282.

Para manter o anonimato e preservar a privacidade dos egressos, foi utilizada uma codificação para cada entrevista, a fim de não restar qualquer ligação entre o conteúdo das entrevistas interpretadas e os participantes. Assim, foram atribuídos os códigos A1, A2, A3, B1, B2 e B3, e assim sucessivamente, de acordo com a ordem cronológica do preenchimento das entrevistas.

\section{Resultados}

De acordo com a análise do software IRAMUTEQ, ao se abordar a temática conhecimento na graduação, percebeu-se a prevalência dos seguintes vocábulos, mencionados de maneira recorrente pelos egressos, de ambas as universidades: "prática", "residência", "conhecimento" e trabalho". Seguidamente, as principais correlações obtidas a partir da análise da Rede de Palavras foram: graduação, prática e residência; conhecimento, prática e vivência; buscar e informação. Os dados apresentados tornaram evidente a importância dos aspectos práticos na formação e de sua associação com o conhecimento acadêmico, além de relacionarem a modalidade de residência neste processo de ensino em serviço.

Cabe pontuar que o levantamento de dados realizado com os egressos sinalizou um ponto de vista mais negativo, trazendo de maneira mais contundente as fragilidades da formação para o contexto do mundo do trabalho, aparecendo com menor frequência a questão das potencialidades deste contexto. Sendo assim, a partir da análise minuciosa da Rede de Palavras em conjunto com leituras do conteúdo das entrevistas, obteve-se duas grandes temáticas a serem discutidas sequencialmente: as fragilidades da formação e a importância das vivências práticas.

Grande parte dos egressos considerou a formação insuficiente para o enfrentamento do mundo do trabalho, correlacionando este ponto ao déficit de vivências práticas experimentadas ao longo da graduação, sendo mencionado por nove (43\%) participantes da Universidade A e sete (32\%) da Universidade B. Os recortes das falas apresentadas a seguir caracterizam esse resultado:

(...) a minha universidade não me preparou para gerenciar a equipe, para coordenar e nem administrar nada. Temos também as questões que envolvem o cuidado, que foram deficitárias. (...) quantas coisas eu vim aprender depois de 
formada, na vivência, porque a instituição, infelizmente, deixou a desejar (A9).

Eu acho que a Universidade nos dá uma base, conhecimento e postura. Acredito que são coisas que não mudam dentro de nós. Mas eu acho que a vivência profissional mesmo não é ensinada (B4).

Os egressos também citaram que, mesmo com as vivências de estágio, a oportunidade da prática era restrita, por entenderem que o quantitativo de estudantes era desproporcional ao número de procedimentos oferecidos nos locais de estágios. Além disso, salientaram que, em alguns casos, a oferta de campos de estágios em hospitais públicos era restrita. As falas destacadas a seguir exemplificam este resultado:

Muitas vezes, nem todo mundo conseguia realizar os procedimentos durante o estágio e às vezes a oferta de campo de estágio era restrita e pouco diversificada (A10).

Eu me formei sem ter passado uma sonda vesical. Só cheguei a realizar esse procedimento na residência. Também não tive prática de punção, porque não tive tantas oportunidades ao longo da graduação (B23).

Como tática capaz de suprir o suposto déficit de conhecimento prático vinculado ao teórico nas universidades, sete (33\%) egressos da Universidade A e quatro (18\%) da Universidade B mencionaram ter recorrido ao programa de residência, como uma estratégia capaz de proporcionar maiores experiências e preparo para o enfrentamento do mundo do trabalho em saúde. A fim de caracterizar tal análise foram apresentados a seguir conteúdos expostos pelos participantes:

Eu me considerei mais preparada depois que eu passei pela residência (...). Assim, quando saí da faculdade ingressei na residência, aí eu vi que não sabia nada (A10).

Eu precisava me sentir capacitada para desenvolver procedimentos, pois sentia que não sabia, que não tinha destreza manual, então eu fui fazer residência por conta disso (B14).

Corroborando, apesar de terem se apresentado em menor frequência, outras fragilidades da formação foram mencionadas, ressaltando-se o medo e a insegurança que assolam o recém-graduado para o enfrentamento do mundo do trabalho:

Tive pouco tempo para realmente ter segurança e me sentir apta para trabalhar. Mas, confesso, bateu um receio, um medo para encarar o trabalho de ser enfermeiro (A18).

Olha, eu confesso que saí com certa insegurança, eu não me sentia pronta em assumir uma atividade assistencial numa unidade de saúde (A20).

Outra limitação do curso de graduação apontada pelos egressos foi a simplista visão da realidade do trabalho, referente não só às atribuições objetivas dos enfermeiros, mas também às questões que envolvem as vivências subjetivas, conforme observado na fala destacada a seguir:

Faltou mais prática (...) Prática, realidade, noção de realidade do trabalho, meandros que envolvem o trabalho do tipo: como lidar com os médicos, técnicos de enfermagem, familiares, pacientes à beira da morte (B10). 
Quando você começa a viver o trabalho, é muito diferente da realidade da faculdade, é muito mais profundo e complexo, envolve muita coisa, muito conhecimento, muita vivência (B27).

A incipiente construção do pensamento clínico ampliado também foi igualmente apontada por eles:

Tinha uma deficiência na técnica e na contextualização da assistência em relação à condição clínica com a parte farmacológica, com o prognóstico do paciente (B25).

Era urgente que minha formação fosse mais crítica e política, e não tive acesso a isso na universidade (B28).

Por outro lado, vale ressaltar que alguns egressos sinalizaram o posicionamento de que a graduação é um processo introdutório da capacitação do indivíduo para o mundo do trabalho, no qual as experiências, vivências e práticas são adquiridas ao longo de sua atuação e enfrentamento. Neste sentido, indicaram que o aprimoramento também depende do próprio profissional, que está em constante aprendizagem.

A vivência profunda do seu trabalho, você só adquire realmente quando somos realmente profissionais, desenvolvendo nossas atividades (A7).

Acho que a universidade é sempre a sua base formadora, porém não deve dar toda a formação necessária. É necessário ir em busca do mais (...). A universidade abre as suas portas para ir em busca de mais conhecimentos, dando essa amplitude de visão de querer buscar mais conhecimentos. Faculdade é só um começo do seu conhecimento. Tem que ir em busca do mais (A14).

\section{Discussão}

As instituições de ensino superior desempenham papel fundamental na formação dos futuros enfermeiros que irão se inserir no mercado, demonstrando que seus egressos devem adquirir a capacidade de utilizar uma diversidade de conhecimentos na solução de problemas do cotidiano. Por essa razão, é imprescindível considerar que a maneira como o conhecimento vem sendo processado pelos profissionais de saúde, em particular a enfermagem, é importante parâmetro para as instituições formadoras. Essas instituições, por sua vez, formam indivíduos para atender não apenas ao mercado de trabalho, mas também às demandas sociais e de saúde e, principalmente, para contribuir com a transformação da sociedade (L. M. Silva et al., 2019; Chrizostimo \& Brandão, 2015).

Considera-se que, na formação do enfermeiro, a construção da prática associada aos conhecimentos teóricos desenvolvidos ao longo do curso de graduação deve capacitar o estudante a atuar com excelência na dinâmica da realidade laboral e da produção dos serviços de saúde (Correa, 2015; Mattia, Kleba, \& Prado, 2018). No entanto, ainda que exista o entendimento de que a formação está pautada em projetos pedagógicos apoiados no desenvolvimento de metodologias dialógicas e participativas, comprometidas com a construção do saber, e que busquem proporcionar ao discente o saber, o saber-fazer e o saber-ser, para atuar com autonomia, eficiência e eficácia nos serviços de saúde (Winters, Prado, \& Heidemann, 2016), observase um hiato entre o discurso construído e o conteúdo experimentado. Isto se traduziu na insatisfação dos egressos, evidenciada através da sinalização de um déficit de vivências práticas ao longo da graduação, ponto de vista que se destacou entre os fatores que levaram os participantes a considerarem a formação universitária frágil.

Esse distanciamento entre o conhecimento apreendido na academia e a realidade vivenciada no espaço laboral pode ser explicado, em parte, pela premissa de que a organização do trabalho é pensada por uns e executada por outros. Há, assim, um choque entre as idealizações dos recém-formados e a necessidade de produção do serviço (Dejours, 2007). Ademais, o mundo 
do trabalho em saúde é dinâmico e contexto de aplicação de uma elevada produção de conhecimentos e tecnologias, onde há necessidade de capacitação contínua e diversificada. Os currículos de graduação não dão conta dessa dinamicidade havendo, assim, certa defasagem entre o que se ensina na academia e o que se vivencia na realidade do trabalho (Silva et al., 2019).

Há também que se aludir à questão do currículo oculto e do currículo oficial pois, apesar do projeto pedagógico e as ementas estarem contidos na filosofia do curso, nas estratégias de ensino e nos conteúdos a serem desenvolvidos, sabe-se que o processo ensino-aprendizagem sofre influência direta da visão de mundo do professor, suas crenças e valores. Estes podem alterar o desenvolvimento das disciplinas, levando à supressão e/ou ao acréscimo de conteúdos no processo de aprendizagem que não necessariamente estão em consonância com o projeto pedagógico dos cursos.

Nesse sentido, enfatizam-se, também, os egressos que recorreram ao programa de residência, compreendendo esta modalidade de ensino em serviço como uma maneira de aperfeiçoar suas práticas nos métodos de aprendizagem ativos e críticos, valorizando o aprender, a partir de situações decorrentes do cotidiano laboral. Assim, a reflexão sobre os problemas corriqueiros permite que o indivíduo busque os fatores causadores e a suposição de soluções (Mattia et al., 2018). Pressupõe-se ainda que a residência possibilite ao enfermeiro recém-graduado vivenciar essa realidade, amadurecendo suas ações e percepções, e transformando seus conhecimentos em atitudes e intervenções.

Outras fragilidades da formação foram mencionadas ao longo das entrevistas, com destaque para o medo e a insegurança que assolam o recém-graduado para o enfrentamento do mundo do trabalho, as idealizações em relação ao cotidiano da profissão, envolvendo aspectos objetivos e subjetivos do serviço, e a dificuldade em elaborar o pensamento clínico ampliado para a prestação do cuidado de qualidade.

As pressões dos serviços de saúde sobre os enfermeiros recém-formados são expressivas, principalmente quando a organização do trabalho os designa para os postos de chefia. Frequentemente, os recém-formados passam a liderar técnicos e auxiliares de enfermagem que apresentam larga experiência e habilidades práticas, o que agrava ainda mais o seu sentimento de insegurança e de despreparo para o enfrentamento da tal realidade (Marques, Santos, Petersen, \& Fidauza, 2018).

Além disso, viver o real mundo do trabalho pode ser tenso e amedrontador pois, durante a graduação, há um docente supervisor que segue apoiando o desenvolvimento dos procedimentos e ações de enfermagem, que assume a responsabilidade sobre o fazer dos estudantes, mas que não mais se encontra no cotidiano laboral. Assim, o professor atua impedindo erros nas realizações de condutas e procedimentos, além de auxiliar no enfrentamento dos conflitos próprios do processo de trabalho em saúde.

Quanto à incipiente visão da realidade do trabalho mencionada pelos egressos, pode-se afirmar que a formação do enfermeiro vai muito além de habilidade psicomotora, destreza manual e conhecimento de princípios científicos. Antes, ela permeia questões que envolvem lidar com pessoas em situações de dor e sofrimento, encarar as relações de poder e hierarquia rígida, enfrentar as condições de riscos ocupacionais do processo laboral, entre outras situações que demandam reflexão, crítica e articulação de conhecimentos que extrapolam o saber biomédico (Chrizostimo \& Brandão, 2015).

Há a necessidade de qualificação para o enfrentamento das adversidades da realidade laboral, considerando aspectos objetivos e subjetivos que transpassam a atuação do enfermeiro no mundo do trabalho em saúde. Há de se ter várias estratégias de ensino-aprendizagem, filosofia e metodologias que respaldem o desenvolvimento das disciplinas curriculares, de forma que as instituições de ensino invistam em uma formação voltada para a realidade prática e o desenvolvimento de profissionais críticos, criativos e transformadores.

A elementar construção do pensamento clínico ampliado, também apontada como fragilidade da formação do enfermeiro, pode ser decorrente do contato, durante a formação, com práticas assistenciais em uma perspectiva "micro", estudando e praticando o cuidado de um ou mais indivíduos, não havendo envolvimento com questões "macro", como políticas 
institucionais e suas inter-relações. Essa complexidade só é percebida após a inserção do egresso na realidade do mundo do trabalho, em que relações conflituosas, lutas políticas e visões gerenciais são sentidas e vivenciadas (Viana et al., 2016).

Em contrapartida, houve alguns egressos que salientaram que a graduação é um processo introdutório da capacitação do indivíduo para o mundo do trabalho. Corrobora-se que há a necessidade de se dar continuidade a esse processo de aprendizagem após a formação na graduação, destacando-se a importância da educação permanente como instrumento para novas possibilidades de atuação no mundo de trabalho, e também como estratégia fundamental para a mudança de realidades (Mattia et al., 2018).

A construção e a consolidação do conhecimento perpassam o ensino da teoria e da prática nas academias; nesse contexto, o indivíduo precisa também se responsabilizar por sua aprendizagem, por meio da inter-relação com outros sujeitos, da prática social e da vivência em realidades distintas (Machado et al., 2019).

Ademais, faz-se necessário aplicar a problematização no ensino prático em saúde, fazendo com que o profissional transforme o olhar curioso do senso comum em um ato contextualizado, avançando de maneira qualitativa no processo de formação, não apenas como profissional, mas também como ser humano. Visando à assistência transformadora, faz-se mister a construção de um indivíduo trabalhador com perfil construtivo, criativo, questionador, interessado e ativo, que seja atuante para transformar os diversos cenários de sua realidade e empenhado na melhoria do cuidar em saúde (Mattia et al., 2018).

\section{Conclusão}

A inserção do ideário neoliberal na área da saúde e a crescente introdução de tecnologias diferenciadas alteraram a configuração do mundo do trabalho, tornando-o insólito para professores e estudantes, sobretudo para o processo de formação em enfermagem. $\mathrm{O}$ trabalho tornou-se ainda mais dinâmico, conflituoso e com demandas que se distanciam do idealizado nos currículos e projetos pedagógicos. Esse contexto laboral causa estranhamentos aos egressos e, por sua vez, conflitos subjetivos e objetivos, resultando em sentimento de capacitação incipiente para enfrentarem as demandas do trabalho a partir do que foi desenvolvido no seu processo de formação. Nesse sentido, salienta-se que tais estranhamentos emergem por conta também do distanciamento entre o que se aprende nas academias e o que é encontrado no mundo real do trabalho.

Nessa perspectiva, o estudo demonstrou que a relação teoria-prática precisa ser fortalecida no ensino de graduação, devendo acontecer de maneira articulada, por meio de sucessivas aproximações com a realidade laboral dos profissionais de enfermagem, indo num crescente de vivências que permitam aprofundar as experiências do que é ser enfermeiro. A teoria deve respaldar a prática, mas também a prática deve retroalimentar a teoria. Esse procedimento, além de qualificar o processo educacional, minimiza o distanciamento entre o que se ensina na teoria e o que se encontra no mundo real do trabalho em saúde.

Numa visão ampliada, sabe-se que é pelo ensino que se modifica a prática assistencial e, por sua vez, o que se vive no mundo do trabalho pode transformar o processo de formação. Ao privilegiar a visão dos egressos, busca-se fortalecer o perfil de enfermeiros críticos e com maiores possibilidades de mudar cenários obsoletos e estranhos para os trabalhadores de enfermagem. Para tal, é imprescindível um esforço sistemático por parte das instituições de ensino, por uma maior aproximação com a realidade do mundo do trabalho, por meio de um currículo problematizador que valorize as vivências práticas, a concretude dos fatos e a correlação do conteúdo teórico com a prática.

É necessário, entretanto, salientar que por mais próximas que sejam a formação profissional e o mundo do trabalho, há conhecimentos que o egresso só adquire pelas experiências vivenciadas no real do trabalho. Um profissional bem preparado técnica e cientificamente depende tanto da academia quanto da capacidade individual e da experiência viva do trabalho. Sendo assim, considera-se que o conhecimento advém tanto da formação profissional quanto do próprio mundo do trabalho, além de que o profissional deve considerar-se sempre em constante e permanente aprendizado. 


\section{Referências}

Alves, I. S. G., \& Silva, J. M. O. (2019). Vivência de uma acadêmica de enfermagem durante o estágio supervisionado na maternidade de alto risco. GepNews, 2(2), 218-223 http://www.seer.ufal.br/index.php/gepnews/article/viewFile/7903/5741

Chrizostimo, M. M., \& Brandão, A. A. P. (2015). A formação profissional do enfermeiro: 'estado da arte'. Enfermería Global, (40),430-445. http://revistas.um.es/eglobal/article/viewFile/208841/181721

Correa, G. C. (2015). Definição e desenvolvimento de competências: um paradigma no processo estratégico. Estudos do CEPE, 39(67),103-116. http://dx.doi.org/10.17058/cepe.v0i41.6294

Costa, M. C. G., Francisco, A. M., \& Hamamoto, C. G. (2019, junho). Metodologia ativa e currículo: uma avaliação dos egressos de um curso de Enfermagem. Atas do Congresso Ibero-Americano em Investigação Qualitativa - CIAIQ. https://proceedings.ciaiq.org/index.php/CIAIQ2019/article/view/2185

Dejours, C. (2007). Psicodinâmica do trabalho na pós-modernidade. In A. M. Mendes, S. C. C. Lima, \& E. P. Facas (Orgs), Diálogos em psicodinâmica do trabalho (pp. 13-26). Brasília: Paralelo 15.

Fettermann, F. A., Nietsche, E. A., Terra, M.G., Salbego, C., Torres, O. M., \& Ramos, T. K. (2018). VER-SUS project: Influences on the training and performance of nurses. Revista Brasileira de Enfermagem, 71(6):2922-2929. http://dx.doi.org/10.1590/0034-7167-2017-0868

Justo, A. M., \& Camargo, B. V. (2014). Estudos qualitativos e o uso de softwares para análises lexicais. In C. Novikoff, S. R. M. Santos, \& O. B. Mithidieri (Orgs.). Caderno de artigos: X SIAT \& II Serpro. Rio de Janeiro: Unigranrio.

Kami, M. T. M., Larocca, L. M., Chaves, M. M. N., Lowen, I. M. V., Souza, V. M. P., \& Goto, D. Y. N. (2016). Trabalho no consultório na rua: uso do software IRAMUTEQ no apoio à pesquisa qualitativa. Escola Anna Nery, 20(3),e20160069. http://dx.doi.org/10.5935/1414-8145.20160069

Machado, V. B., Antonio, P. S., Andrade, J., Bolina, A. F., Santos, P. H. F., \& Paranagua, T. T. B. (2019, junho). Percepção dos docentes acerca das competências desenvolvidas pelos egressos de enfermagem por meio do ensino simulado: um estudo qualitativo. Atas do Congresso Ibero-Americano em Investigação Qualitativa - CIAIQ. Lisboa, Portugal, 8. https://repositorio.unb.br/bitstream/10482/35185/1/ARTIGO_PercepcaoDocentesAcercaCompetencias.pdf

Marques, M., Santos, D. F., Petersen, M. E. O., \& Fidauza, M. G. (2018). A importância da educação continuada na socialização do novo profissional de enfermagem. Revista Inova Saúde, 8(2). http://dx.doi.org/10.18616/inova.v8i2.2468

Mattia, B. J., Kleba, M. E., \& Prado, M. L. (2018). Nursing training and professional practice: an integrative review of literature. Revista Brasileira de Enfermagem, 71(4),2039-2049. http://dx.doi.org/10.1590/0034-7167-2016-0504

Püschel, V. A. A., Costa, D., Reis, P.P., Oliveira, L. B., \& Carbogim, F.C. (2017). Nurses in the labor market: professional insertion, competencies and skills. Revista Brasileira de Enfermagem, 70(6),1220-1226. http://dx.doi.org/10.1590/0034-7167-2016-0061

Silva, B. A., Peres, C. R. F. B., Pio, D. A. M., Marin, M. J. S., \& Otani, M. A. P. (2019). Estudantes de enfermagem e medicina inseridos na prática profissional: percepção de equipes. Revista Psicologia, Diversidade e Saúde, 8(3),298-308http://dx.doi.org/10.17267/2317-3394rpds.v8i3.2395

Silva, L. M., Santana, T. C. P., Silva, L. R. F. G., Rocha, L. M., Canhoto, C. T.S., Dantas, K. L., Silva, A. C. F. A., Silva, E. V., Melo, M. I. B., Silva, R. M., Figueira, M. C. S., Siqueira, R. M., Martins, M. R. R., Silva, S. L., \& Oliveira, C. R. (2019). Estágio curricular supervisionado: dificuldades e perspectivas vivenciadas por acadêmicos de enfermagem. Revista Eletrônica Acervo Saúde, (18),e662. https://doi.org/10.25248/reas.e662.2019

Souza, M. A. R., Wall, M. L., Thuler, A. C. M. C., Lowen, I. M. V., \& Peres, A. M. (2018). O uso do software IRAMUTEQ na análise de dados em pesquisas qualitativas. Revista da Escola de Enfermagem da USP, 52,e03353. http://dx.doi.org/10.1590/S1980-220X2017015003353

Viana, M. A. S., Fortuna, C. M., Borges, F. A., Gatto, J. R., Jr., Matumoto, S., \& Clapis, M. J. (2016). Analysis of attitudinal assessment in the education of mid-level nursing professionals. Revista Eletrônica de Enfermagem, 18,e1200. http://dx.doi.org/10.5216/ree.v18.41632

Winters, J. R. F., Prado, M. L., \& Heidemann, I. T. S. B. (2016). A formação em enfermagem orientada aos princípios do Sistema Único de Saúde: percepção dos formandos. Escola Anna Nery, 20(2),248-253. https://doi.org/10.5935/1414-8145.20160033

Zhou, W., He, G., Wang, H., He, Y., Yuan, Q., \& Liu, D. (2015). Job dissatisfaction and burnout of nurses in Hunan, China: A cross-sectional survey. Nursing \& Health Sciences, 17(4),444-450. https://doi.org/10.1111/nhs.12213 